\title{
Dopamine receptor D2 is correlated with gastric cancer prognosis
}

\author{
JIASHENG MU ${ }^{1,2^{*}}$, WEIDAN HUANG ${ }^{3 *}$, ZHUJUN TAN $^{1,2}$, MAOLAN LI $^{1,2}$, LIN ZHANG $^{1,2}$, QICHEN DING $^{1,2}$, \\ XIANGSONG WU ${ }^{1,2}$, JIANHUA LU ${ }^{1,2}$, YINGBIN LIU ${ }^{1,2}$, QIAN DONG ${ }^{1,2}$ and HAINENG XU ${ }^{4}$ \\ ${ }^{1}$ Department of General Surgery, Xinhua Hospital Affiliated to Shanghai Jiao Tong University School of Medicine; \\ ${ }^{2}$ Institute of Biliary Tract Disease, Shanghai Jiao Tong University School of Medicine, Shanghai 200092; \\ ${ }^{3}$ State Key Laboratory of Cell Biology, Institute of Biochemistry and Cell Biology, Shanghai Institutes for Biological Sciences, \\ Chinese Academy of Sciences, Shanghai 200031, P.R. China; ${ }^{4}$ Department of Radiation Oncology, \\ University of Pennsylvania School of Medicine, Philadelphia, PA 19104, USA
}

Received July 23, 2015; Accepted November 3, 2016

DOI: $10.3892 / 01.2017 .5573$

\begin{abstract}
It has been reported previously that a dopamine receptor D2 (DRD2) antagonist was able to induce cancer cell apoptosis and that DRD2 was expressed at high levels in pituitary adenomas. However, the expression of DRD2 in gastric cancer and its correlation with the prognosis of patients with gastric cancer remain to be elucidated. In the present study, the expression of DRD2 in 84 paired gastric cancer tissues and respective adjacent non-cancerous tissues were detected using an immunohistochemical assay. The correlation between the expression of DRD2 and the with survival durations of the patients with gastric cancer was analyzed using Kaplan-Meier analysis. In addition, online resources were utilized to further analyze the correlation between the mRNA expression level of DRD2 and prognosis. The effect of the DRD2 antagonist, thioridazine, on the proliferation of the AGS gastric cancer cells was determined. The results of the present study showed that the percentage of gastric cancer cases with a high expression level of DRD2 (51.2\%) was higher, compared with that of cases with a low expression level of DRD2 (39.3\%). Patients with a higher expression of DRD2 had shorter survival durations. The online database analysis revealed that the expression of DRD2 was also inversely correlated with the prognosis
\end{abstract}

Correspondence to: Dr Haineng Xu, Department of Radiation Oncology, University of Pennsylvania School of Medicine, 3400 Civic Center Blvd., Philadelphia, PA 19104, USA

E-mail: xuhaineng123@163.com

Dr Qian Dong, Department of General Surgery, Xinhua Hospital Affiliated to Shanghai Jiao Tong University School of Medicine, 1665 Kong-Jiang Road, Shanghai 200092, P.R. China

E-mail: dongqianqian71@163.com

*Contributed equally

Key words: dopamine receptor, dopamine receptor D2, gastric cancer, thioridazine, online database of patients with gastric cancer. Furthermore, the DRD2 antagonist, thioridazine, inhibited the growth of AGS gastric cancer cells. In conclusion, as the expression of DRD2 was negatively correlated with survival durations in patients with gastric cancer, it may be considered as a prognosis marker in the future. Developing DRD2 antagonists may assist in increasing the efficiency of cancer therapy.

\section{Introduction}

Gastric cancer is one of the most common types of cancer and ranks as the second leading cause of cancer-associated mortality worldwide (1-3), with the majority of patients residing in China. Due to the non-specific symptoms of gastric cancer at early stages, the majority of patients present at an advanced stage at initial diagnosis, making cancer therapy challenging. At present, traditional methods, including surgery, chemotherapy and radiation therapy, are the major approaches used to treat gastric cancer $(4,5)$. However, the drugs in current use have limited efficiency due to the advanced stages of cancer. There is an urgent requirement to identify suitable targets for cancer therapy and to develop an efficient drug.

Dopamine receptors are a class of G-protein-coupled receptors and are important in the nervous system. Dopamine receptors are involved in various neurological processes, with dopamine as their ligand. Disordered dopamine secretion or inactivation of dopamine receptors may lead to various neurological diseases, including Parkinson's disease, schizophrenia and social phobia (6-8). In this class of receptors, dopamine receptor D2 (DRD2) is an important member.

In previous years, in addition to its traditional function, DRD2 has been reported to be correlated with pituitary tumors $(9,10)$. A study investigating gene polymorphisms in DRD2 found that DRD2 alleles are associated with tobacco use and lung cancer $(11,12)$. Genome-wide short hairpin RNA screens have supported the importance of DRD2 in glioblastoma. The mRNA and protein expression levels of DRD2 were found to be elevated in clinical glioblastoma specimens, compared with matched non-tumor tissues, and the inhibition of DRD2 and epidermal growth factor receptor induced 
synergistic antitumor activity (11). A correlation between the expression of DRD2 and tumors has also been found in bladder cancer and leukemia $(13,14)$. These findings suggest an association between DRD2 and cancer, and its potential for use as a potential target for cancer therapy. A small molecule screen of targeting cancer stem cells demonstrated that the DRD2 inhibitor, thioridazine, showed potent antileukemic stem cell activity (15). As cancer stem cells are a subpopulation of cells responsible for cancer progression, metastasis and chemoresistance (16-18), this finding further supported the potential significant function of DRD2 in cancer. Although the inhibition of DRD2 has been reported in several studies, to the best of our knowledge, the correlation between the expression of DRD2 in gastric cancer and survival durations has not been reported. In the present study, 84 gastric cancerous tissue samples and matched adjacent non-tumor tissue samples were collected. The protein expression levels of DRD2 were detected, and the correlation between the expression of DRD2 and prognosis was analyzed. The aim of the present study aimed to identify a marker for gastric cancer prognosis and develop a potential target for gastric cancer therapy.

\section{Materials and methods}

Sample collection and tissue chip processing. A total of 84 gastric cancerous tissues and respective adjacent non-cancerous tissues were obtained from 84 patients who were diagnosed with cancer and underwent surgical resection in the Department of General Surgery, Xinhua Hospital affiliated with Shanghai Jiaotong University (Shanghai, China) between February 2007 and May 2008. The tissue samples were from 64 men and 20 women, who were aged between 34 and 83 years. The majority of the patients $(81 / 84)$ were diagnosed without metastasis. According to the World Health Organization classification, 34 cases were defined as well- or moderately differentiated, and the other 50 cases were considered to be poorly differentiated. In terms of tumor-node-metastasis (TNM) classification, 32 cases were classified as early or median stage and 52 cases were classified as late stage. Informed consent was obtained prior to surgery. All experiments using human tissues were performed in accordance with the Institutional Review Board of Shanghai Jiaotong University. The samples were observed by pathologists, and those suitable for experiments were selected and processed into a paraffin-embedded tissue chip by Shanghai Superchip Biotechnology Corporation (Shanghai, China).

Immunohistochemical assay. The expression levels of DRD2 in the gastric cancer tissues and adjacent non-cancerous tissues were detected using an immunohistochemical assay. The paraffin-embedded tissues were preheated at $63^{\circ} \mathrm{C}$ for $1 \mathrm{~h}$, and then deparaffinized and rehydrated using dimethylbenzene and degrading ethanol solution. Following rinsing with water three times, the tissues were incubated in citrate buffer (prepared by mixing $82 \mathrm{ml} 0.1 \mathrm{M}$ sodium citrate, $18 \mathrm{ml} 0.1 \mathrm{M}$ citric acid and $900 \mathrm{ml}$ distilled water), heated in the autoclave for $5 \mathrm{~min}$ and cooled to room temperature for $30 \mathrm{~min}$. Endogenous peroxidase activity was quenched using methanol and hydrogen peroxidase solution (38.4 ml methanol,
$12 \mathrm{ml} \mathrm{30 \%} \mathrm{hydrogen} \mathrm{peroxidase} \mathrm{and} 9.6 \mathrm{ml}$ distilled water) for $15 \mathrm{~min}$ at room temperature. Following washing with phosphate-buffered saline (PBS) three times, the tissues were incubated with mouse anti-DRD2 monoclonal antibody (Santa Cruz Biotechnology, Inc., Santa Cruz, CA, USA) at a dilution of 1:100 in a humidified atmosphere. Following staining with the primary antibody at $4^{\circ} \mathrm{C}$ overnight, the tissues were incubated with secondary antibody from the mouse EnVision ${ }^{\mathrm{TM}+} / \mathrm{HRP}$ kit (Dako North America, Inc., Carpinteria, CA, USA) for $1 \mathrm{~h}$ at room temperature, following which they were reacted with DAB solution (Dako North America, Inc.) for $5 \mathrm{~min}$ at room temperature. The tissues were then stained with hematoxylin (Sigma-Aldrich; Merck Millipore, Darmstadt, Germany) for $40 \mathrm{sec}$ and subsequently with $0.25 \%$ hydrochloric acid alcohol solution for $2 \mathrm{sec}$. The tissues were then dehydrated with graded ethanol and dimethylbenzene, mounted and visualized under a microscope (Leica BM E microscope; Leica Biosystems Inc., Buffalo Grove, IL, USA).

The expression levels of DRD2 in the tissues were evaluated by two independent examiners who were blinded to the patient outcomes and classification of the tissues. The expression levels of DRD2 were determined as the percentage of positive cells and intensity of staining. The intensity of the staining was classified between 0 and 3 (0, none; 1 , weak; 2 , moderate; 3 , strong). The level of DRD2 in each sample was calculated as the labelling intensity $x$ percentage of positive cells. The evaluations of the two observers were identical for the majority of samples. The remaining tissues were reevaluated and the final results were analyzed.

Cell culture. The AGS gastric cancer cells were obtained from the Cell Bank of the Shanghai Institutes for Biological Sciences, Chinese Academy of Sciences (Shanghai, China). The AGS cells were grown in Roswell Park Memorial Institute 1640 medium (Gibco; Thermo Fisher Scientific, Inc., Waltham, MA, USA) containing $10 \%$ fetal bovine serum (Sigma-Aldrich; Merck Millipore) and $1 \mathrm{X}$ penicillin-streptomycin (Sigma-Aldrich; Merck Millipore). The cells were incubated in a humidified atmosphere with $5 \% \mathrm{CO}_{2}$ at a temperature of $37^{\circ} \mathrm{C}$.

Cell survival detection using a 3-(4,5-dimethylthiazol-2-yl)-2, 5-diphenyl tetrazolium bromide (MTT) assay. The AGS cells were seeded into 96-well dishes (Corning Incorporated, Corning, NY, USA) at a density of $1 \times 10^{4}$ cells/well. They were treated with thioridazine (Sigma-Aldrich; Merck Millipore) at indicated concentrations after $12 \mathrm{~h}$.

To the cells, $10 \mu \mathrm{l}$ MTT (4 mg/ml; Beyotime Institute of Biotechnology, Haimen, China) was added $48 \mathrm{~h}$ following treatment and incubated at $37^{\circ} \mathrm{C}$ for $4 \mathrm{~h}$. The precipitate was dissolved in $100 \mu \mathrm{l}$ DMSO following the removal of supernatant. The absorbance at wavelengths of 595 and $630 \mathrm{~nm}$ were measured. The cell viability was calculated as the optical density $(\mathrm{OD})_{595}-\mathrm{OD}_{630}$. Experiments were repeated three times.

Statistical analysis. The overall survival durations of the patients with gastric cancer were analyzed using Kaplan-Meier analysis. The groups were compared using one-way analysis of variance using R 2.9.0 software. The correlation between 
the mRNA levels of DRD2 and overall survival durations in patients with gastric cancer were examined using online database resources and calculated using Kaplan-Meier analysis (http://kmplot.com/analysis) (19).

\section{Results}

Gastric cancer tissues show higher protein expression levels of DRD2, compared with adjacent non-cancerous tissues. The expression levels of DRD2 in gastric cancer tissues and respective adjacent non-cancerous tissues were detected using an immunohistochemical assay. It was found that, in the 84 paired samples, $51.2 \%$ (43/84) of the cancerous tissues showed higher expression of DRD2 (Fig. 1), whereas the percentage of adjacent non-cancerous tissues showing high protein expression levels of DRD2 was only $39.3 \%$ (33/84). The remaining $9.5 \%$ $(8 / 84)$ paired tissues showed similar levels of DRD2 in the cancerous and non-cancerous tissues.

Patients with higher expression levels of DRD2 exhibit shorter survival duration. To determine whether the expression of DRD2 is correlated with the survival durations of the patients, the present study analyzed the association between the level of DRD2 and survival durations using Kaplan-Meier analysis with a log-rank test. The median survival duration of patients with higher expression levels of DRD2 was 28 months, which was shorter, compared with that of the patients with lower expression levels of DRD2, which was $~ 39$ months (Fig. 2A). The correlation between the mRNA levels of DRD2 and prognosis were also analyzed using online resources. Patients with gastric cancer with higher transcription levels of DRD2 also had reduced survival durations (Fig. 2B). This further demonstrated the inverse correlation between the expression of DRD2 and the survival durations of patients with gastric cancer. The correlations between the expression of DRD2 and other clinic pathological features were also analyzed in the present study, however, no significant associations were found between the expression of DRD2 and age, gender, tumor volume, differentiation, metastasis, node positivity or TNM stage in the patients with gastric cancer (Table I).

DRD2 inhibitor, thioridazine, decreases gastric cancer cell survival. To assess whether the DRD2 inhibitor, thioridazine, has any inhibitory effect on gastric cancer cells, the AGS cells were treated with different concentrations of thioridazine and the cell viability was measured $48 \mathrm{~h}$ later. Only $45 \%$ of the AGS cells were viable following treatment with $12 \mu \mathrm{M}$ thioridazine and $10 \%$ of the cells were viable following treatment with $20 \mu \mathrm{M}$ thioridazine (Fig. 3). This demonstrated that the DRD2 inhibitor inhibited the growth of the gastric cancer cells.

\section{Discussion}

The dopamine receptor pathway is of vital importance in the neurological system. Disorder of the dopamine pathway may result in severe neurological disease. Previously, the DRD2 inhibitor thioridazine was predominantly used for patients with psychosis, however, it has been found to have an antagonist
Table I. Correlation between pathological factors and expression of DRD2 in tumor tissues.

\begin{tabular}{|c|c|c|c|}
\hline \multirow[b]{2}{*}{ Variable } & \multicolumn{2}{|c|}{ DRD2 (n) } & \multirow[b]{2}{*}{ P-value } \\
\hline & Low & High & \\
\hline Age & & & 0.578 \\
\hline$\geq 65$ years & 26 & 18 & \\
\hline$<65$ years & 26 & 14 & \\
\hline Gender & & & 0.466 \\
\hline Female & 11 & 9 & \\
\hline Male & 41 & 23 & \\
\hline Tumor volume & & & 0.623 \\
\hline$\geq 35 \mathrm{~cm}^{3}$ & 23 & 16 & \\
\hline$<35 \mathrm{~cm}^{3}$ & 27 & 15 & \\
\hline Tumor differentiation & & & 0.163 \\
\hline I, II, II-III & 18 & 16 & \\
\hline III & 34 & 16 & \\
\hline Metastasis & & & 0.299 \\
\hline Yes & 1 & 2 & \\
\hline No & 51 & 30 & \\
\hline Node positive & & & 0.587 \\
\hline$\geq 5$ & 25 & 13 & \\
\hline$<5$ & 27 & 18 & \\
\hline TNM stage & & & 0.311 \\
\hline $1 \mathrm{~A}-2 \mathrm{~B}$ & 22 & 10 & \\
\hline $3 \mathrm{~A}-4$ & 30 & 22 & \\
\hline
\end{tabular}

DRD2, dopamine receptor D2; TNM, tumor-node-metastasis.

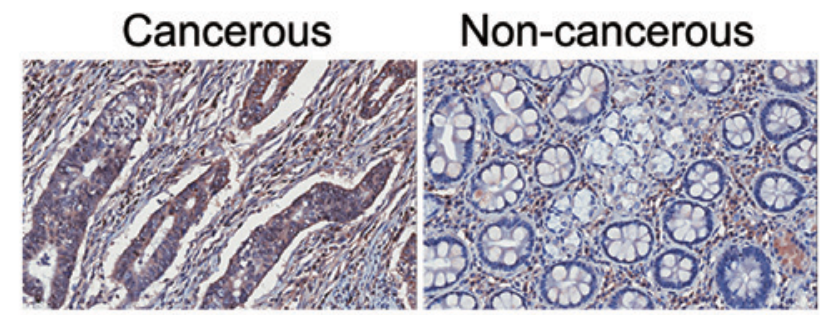

Figure 1. Gastric cancer tissues show higher protein expression levels of DRD2, compared with adjacent non-cancerous tissues. The expression levels of DRD2 was scored in gastric cancer tissues (left) and respective non-cancerous tissues (right). Original magnification, x100. DRD2, dopamine receptor D2.

effect in tumors. Thioridazine has shown anti-angiogenic effects and apoptosis-inducing abilities in breast and ovarian cancer $(20,21)$. It also induced cell death in cervical and gastric cancer $(22,23)$. These findings indicate that DRD2 may be involved in cancer progression, and that its expression may be correlated with cancer prognosis.

In the present study, 84 pairs of tumor and adjacent non-tumor tissues were collected. Immunochemical analysis was used to detect the expression levels of DRD2 in the tissues. DRD2 was expressed at a higher level in tumors, 
A

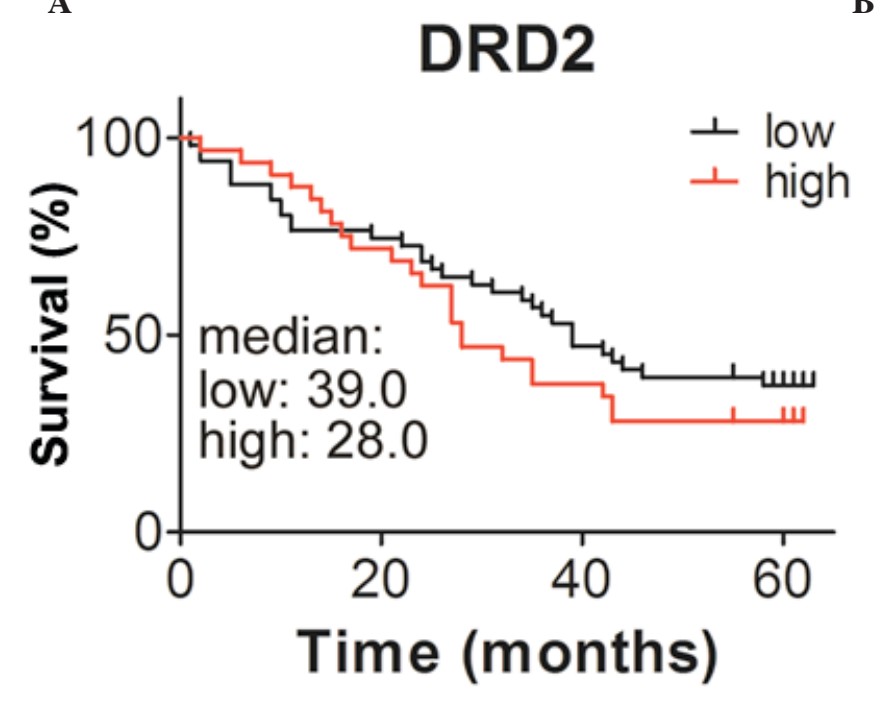

B

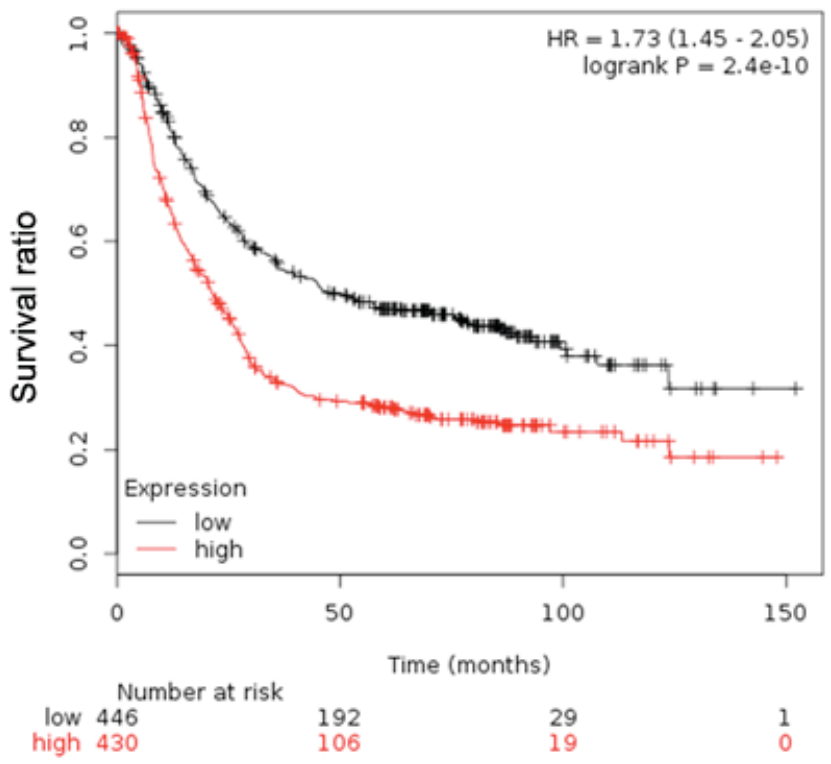

Figure 2. Patients with higher expression levels of DRD2 exhibit shorter survival durations. (A) Correlation between the expression levels of DRD2 and the survival durations of patients with gastric cancer, analyzed using the Kaplan-Meier method. (B) Analysis of the correlation between the survival durations of patients with gastric cancer and the expression levels of DRD2 was performed using the Kaplan-Meier method and online resources. DRD2, dopamine receptor D2.

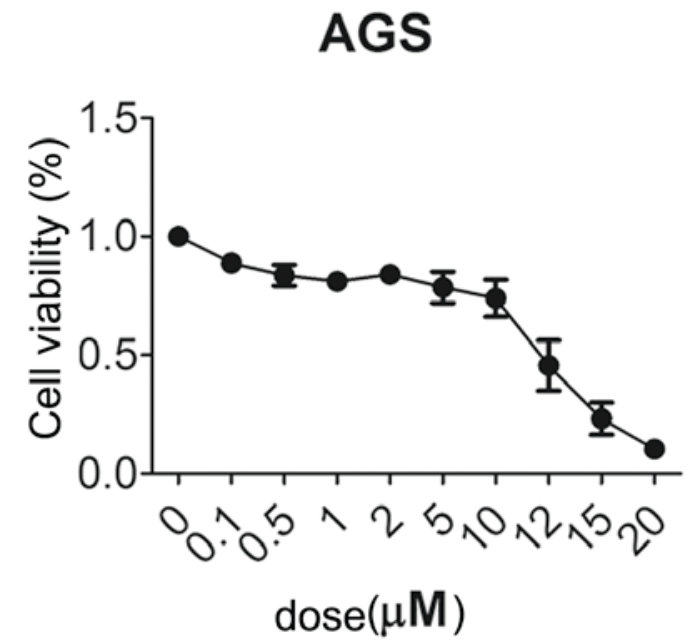

Figure 3. Dopamine receptor D2 inhibitor thioridazine decreases gastric cancer cell survival. The inhibitory effect of thioridazine on AGS cell proliferation was measured using a 3-(4,5-dimethylthiazol-2-yl)-2, 5-diphenyl tetrazolium bromide assay.

compared with adjacent matched non-tumor tissues (Fig. 1). Patients with higher expression levels of DRD2 had lower survival durations (Fig. 2). These results demonstrated that the expression of DRD2 was negatively associated with survival durations of patients with gastric cancer. The inhibition of DRD2 by thioridazine in the gastric cancer cell line markedly inhibited cell growth (Fig. 3). As the expression of DRD2 was correlated with the survival of patients with gastric cancer, it may function in cancer cell survival. Developing small molecule drugs to target DRD2 may provide antitumor effects. Sachlos et al (15) found the anticancer stem cell effects of thioridazine through drug screening. The inhibition of DRD2 by thioridazine showed potent anticancer and cancer stem cell properties. In addition to thioriazine, other DRD2 inhibitors may have inhibitory effects on cancer cells, even cancer stem cells, and require further investigation.

Gastric cancer is one of the most common types of cancer. It is important to identify specific markers, develop efficient drugs and use drug combinations to cure gastric cancer. In addition, it is important to prevent the development of gastric cancer by improving lifestyle factors. Maintaining the freshness of food in a refrigerator, and decreasing the prevalence of Helicobacter pylori by improving hygiene and reducing salt intake may assist in preventing the occurrence of gastric tumors $(2,24)$. Regular detection of Helicobacter pylori may assist in further decreasing the occurrence of gastric cancer. It is hoped that, in the future, the incidence of gastric cancer can be decreased through improved lifestyle. For individuals with gastric cancer, the detection of DRD2 may assist in evaluating prognosis, and the use of DRD2 inhibitors alone or together with other drugs may have potent inhibitory effects on tumors.

\section{Acknowledgements}

This study was supported by the National Natural Science Foundation of China (grant nos. 81172026, 81272402, 81301816 and 81172029), the Foundation of Shanghai Outstanding Academic Leaders (grant no.11XD1403800), the National High Technology Research and Development Program (863 Program; grant no. 2012AA022606), the Post-doctoral Research Foundation of China (grant no. 2012M511107), the Foundation for Interdisciplinary research of Shanghai Jiao Tong University (grant no. YG2011ZD07), the Shanghai Science and Technology Commission Inter-governmental International Cooperation Project (grant no. 12410705900), the Shanghai Science and Technology Commission Medical-guiding Project (grant no. 12401905800) and the Program for Changjiang Scholars and Post-doctoral Research Program of Shanghai (grant no. 12R21415300). 


\section{References}

1. Torre LA, Bray F, Siegel RL, Ferlay J, Lortet-Tieulent J and Jemal A: Global cancer statistics, 2012. CA Cancer J Clin 65: 87-108, 2015.

2. Jemal A, Bray F, Center MM, Ferlay J, Ward E and Forman D: Global cancer statistics. CA Cancer J Clin 61: 69-90, 2011.

3. Parkin DM, Bray F, Ferlay J and Pisani P: Global cancer statistics, 2002. CA Cancer J Clin 55: 74-108, 2005.

4. Takahashi T, Saikawa Y and Kitagawa Y: Gastric cancer: Current status of diagnosis and treatment. Cancers (Basel) 5: 48-63, 2013.

5. Berardi R, Scartozzi M, Romagnoli E, Antognoli S and Cascinu S: Gastric cancer treatment: A systematic review. Oncol Rep 11: 911-916, 2004.

6. Kienast T and Heinz A: Dopamine and the diseased brain. CNS Neurol Disord Drug Targets 5: 109-131, 2006.

7. Fuxe K, Manger P, Genedani S and Agnati L: The nigrostriatal DA pathway and Parkinson's disease. J Neural Transm Suppl 71-83, 2006.

8. Schneier FR, Liebowitz MR, Abi-Dargham A, Zea-Ponce Y, Lin SH and Laruelle M: Low dopamine D(2) receptor binding potential in social phobia. Am J Psychiatry 157: 457-459, 2000.

9. Hentges ST and Low MJ: Ovarian dependence for pituitary tumorigenesis in D2 dopamine receptor-deficient mice. Endocrinology 143: 4536-4543, 2002.

10. Wood DF, Johnston JM and Johnston DG: Dopamine, the dopamine D2 receptor and pituitary tumours. Clin Endocrinol (Oxf) 35: 455-466, 1991.

11. Li J, Zhu S, Kozono D, Ng K, Futalan D, Shen Y, Akers JC, Steed T, Kushwaha D, Schlabach M, et al: Genome-wide shRNA screen revealed integrated mitogenic signaling between dopamine receptor D2 (DRD2) and epidermal growth factor receptor (EGFR) in glioblastoma. Oncotarget 5: 882-893, 2014.

12. Senogles SE: D2 dopamine receptor-mediated antiproliferation in a small cell lung cancer cell line, NCI-H69. Anticancer Drugs 18: 801-807, 2007.

13. Clague J, Cinciripini P, Blalock J, Wu X and Hudmon KS: The D2 dopamine receptor gene and nicotine dependence among bladder cancer patients and controls. Behav Genet 40: 49-58, 2010.
14. Basu B, Sarkar C, Chakroborty D, Ganguly S, Shome S, Dasgupta PS and Basu S: D1 and D2 dopamine receptor-mediated inhibition of activated normal $\mathrm{T}$ cell proliferation is lost in jurkat T leukemic cells. J Biol Chem 285: 27026-27032, 2010.

15. Sachlos E, Risueno RM, Laronde S, Shapovalova Z, Lee JH, Russell J, Malig M, McNicol JD, Fiebig-Comyn A, Graham M, et al: Identification of drugs including a dopamine receptor antagonist that selectively target cancer stem cells. Cell 149: 1284-1297, 2012.

16. Li K, Dan Z and Nie YQ: Gastric cancer stem cells in gastric carcinogenesis, progression, prevention and treatment. World J Gastroenterol 20: 5420-5426, 2014.

17. Abdullah LN and Chow EK: Mechanisms of chemoresistance in cancer stem cells. Clin Transl Med 2: 3, 2013.

18. Li F, Tiede B, Massagué J and Kang Y: Beyond tumorigenesis: Cancer stem cells in metastasis. Cell Res 17: 3-14, 2007.

19. Szász AM, Lánczky A, Nagy Á, Förster S, Hark K, Green JE, Boussioutas A, Busuttil R, Szabó A and Győrffy B: Cross-validation of survival associated biomarkers in gastric cancer using transcriptomic data of 1,065 patients. Oncotarget: Jun 30, 2016 (Epub ahead of print).

20. Byun HJ, Lee JH, Kim BR, Kang S, Dong SM, Park MS, Lee SH, Park SH and Rho SB: Anti-angiogenic effects of thioridazine involving the FAK-mTOR pathway. Microvasc Res 84: 227-234, 2012.

21. Rho SB, Kim BR and Kang S: A gene signature-based approach identifies thioridazine as an inhibitor of phosphatidylinositol-3'-kinase (PI3K)/AKT pathway in ovarian cancer cells. Gynecol Oncol 120: 121-127, 2011.

22. Mu J, Xu H, Yang Y, Huang W, Xiao J, Li M, Tan Z, Ding Q, Zhang L, Lu J, et al: Thioridazine, an antipsychotic drug, elicits potent antitumor effects in gastric cancer. Oncol Rep 31: 2107-2114, 2014.

23. Kang S, Dong SM, Kim BR, Park MS, Trink B, Byun HJ and Rho SB: Thioridazine induces apoptosis by targeting the $\mathrm{PI} 3 \mathrm{~K} / \mathrm{Akt} / \mathrm{mTOR}$ pathway in cervical and endometrial cancer cells. Apoptosis 17: 989-997, 2012.

24. Levi E, Sochacki P, Khoury N, Patel BB and Majumdar AP: Cancer stem cells in Helicobacter pylori infection and aging: Implications for gastric carcinogenesis. World J Gastrointest Pathophysiol 5: 366-372, 2014. 